\title{
Equilibrium shapes of poly-crystalline silicon nanodots
}

\author{
M. D. Korzec * $\quad$ B. Wagner * M. Roczen ${ }^{\dagger} \quad$ M. Schade ${ }^{\ddagger}$ \\ B. Rech ${ }^{\dagger}$
}

Department of Mathematics, TU Berlin, Germany, Preprint 2013/21

August 21, 2013

\begin{abstract}
This study is concerned with the topography of nanostructures consisting of arrays of poly-crystalline nanodots. Guided by transmission electron microscopy (TEM) measurements of c-Si nanodots that evolved from a "dewetting" process of an a-Si layer from a $\mathrm{SiO}_{2}$ coated substrate, we investigate appropriate surface energy density formulations to model these equilibrium geometries. We explore the influence of smooth transitions between the energy density states at grain boundaries on the associated surface morphology of the equilibrium poly-crystals. Furthermore, we introduce a new numerical minimization formulation that allows to account for adhesive energy from an underlying substrate. We demonstrate our approach first for the unbounded case, where the solutions can be compared to well-known Wulff constructions, before we treat the general case for interfacial energy settings that support partial 'wetting'. Eventually, we use the method to study two-dimensional shapes of poly-crystalline silicon nanodots.
\end{abstract}

Keywords: equilibrium shapes, anisotropic surface energy, adhesive energy, constrained optimization, Wulff construction, silicon nanodots, TEM

*Institute of Mathematics, Technische Universität Berlin, Straße des 17. Juni 136, 10623 Berlin, Germany (\{korzec, bwagner\}@math.tu-berlin.de).

${ }^{\dagger}$ Helmholtz-Zentrum Berlin, Institute for Silicon Photovoltaics, Kekuléstraße 5, 12489 Berlin, Germany (\{Maurizio.Roczen, bernd.rech\} @helmholtz-berlin.de).

†Interdisziplinäres Zentrum für Materialwissenschaften, Martin-Luther-Universität HalleWittenberg, Heinrich-Damerow-Str. 4, 06120 Halle, Germany (martin.schade@physik.unihalle.de). 


\section{Introduction}

The morphologies of material surfaces and interfaces are of fundamental importance for material properties. For many nano-technological applications understanding this relationship is the basis for designing materials with specific functional properties. Of interest are the material properties that are induced by patterns of nanodots near their equilibrium. Such patterns arise from a number of processes; for example during the growth of nucleated crystalline silicon grains in a matrix of amorphous silicon, or the epitaxial growth of quantum dots, or after solid dewetting of a thin amorphous or crystalline film from a solid surface. Controlling and optimizing such complex processes to arrive at specific stable patterns is the topic of intensive research, we refer to the recent review by Thompson [21] for an overview and further references.
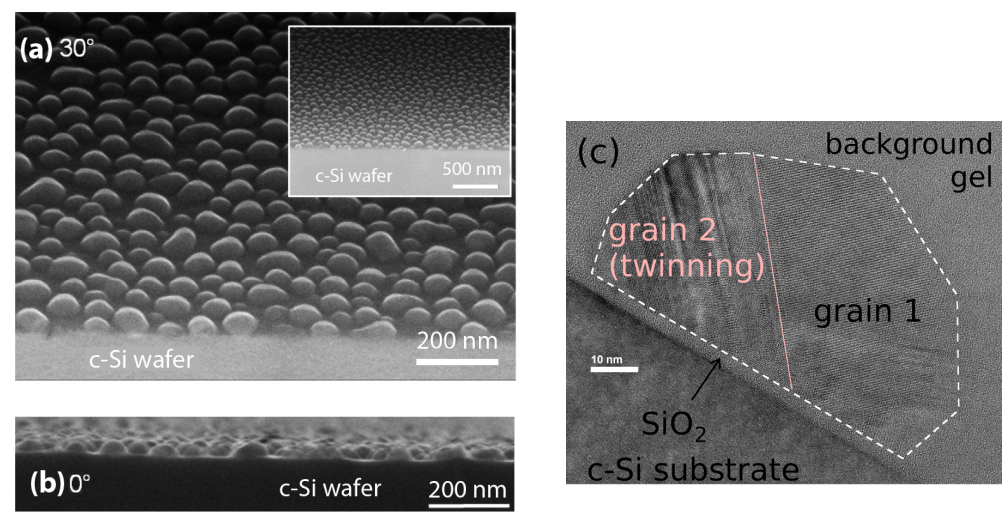

Figure 1: (a) and (b): Angle dependent SEM images of a dewetting undoped Si layer. Initial thickness of the a-Si: $10 \mathrm{~nm}$, temperature $1000^{\circ} \mathrm{C}$; (c) TEM image of poly-crystalline nanodot (interfaces are sketched). Initial thickness of the a-Si: 20nm, temperature $700^{\circ} \mathrm{C}$.

The main focus of our study is the description of such equilibrated patterns, with the specific interest in the formation of crystalline and poly-crystalline nanodots that result during the dewetting and re-crystallization of thin amorphous silicon layers on $\mathrm{SiO}_{2} / \mathrm{Si}(111)$ substrates $[23,22,13,15]$. An example is depicted in Figure 1 using electron microscope imaging. Dependent on the initial thickness of the a-Si layer, temperature and annealing time, the layout of the nanodots changes. One particular such array is shown from two different angles in (a) and (b). In Figure 1 (c) a larger dot, formed from an initially thicker a-Si layer (20nm), is shown in a TEM image. Two grains are indicated by the sketched lines, leading 
to a faceted poly-crystalline nanodot. One can see a dark-bright tessellation in the left grain, which appears due to twinning, which is well-known from bulk a-Si recrystallization, see for example Batstone [2].
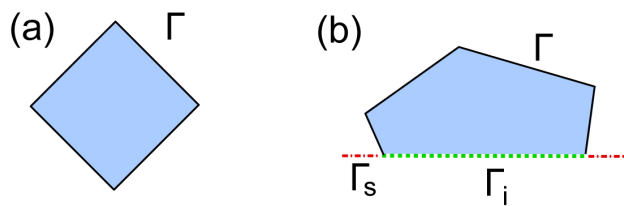

Figure 2: (a) Sketch of an unbounded anisotropic 2D equilibrium shape; (b) Setting for an anisotropic nanodot located on a rigid substrate.

Any realistic mathematical model for c-Si nanodot formation needs to account for the influence of anisotropic surface energy densities. This is clearly demonstrated during the dewetting process of c-Si from $\mathrm{SiO}_{2}$. It is shown in [12] that the dewetting dynamics is dependent on the orientation of artificially created edges in the thin film. It has further been shown that the equilibrium shapes of nanodots that are created by c-Si annealing have a distinct faceted dome structured equilibrium shape $[1,12]$. As the anisotropy is already crucial in one-crystalline layers, it also plays a major role in the re-crystallization and dewetting of thin a-Si films. In order to describe and calculate anisotropic geometries of poly-crystalline nanodots attached to a substrate, it is essential to consider an adhesive energy with the substrate and neighboring grain boundaries.

We begin our derivation of a continuum description that includes these effects with the fundamental problem of the energy minimization task

$$
\min _{\Omega} \int_{\partial \Omega} \gamma d S \text { s.t. }|\Omega|=\text { const . }
$$

Its solution yields an equilibrium shape with minimal surface energy that has a prescribed volume. In Figure 2 (a) such a geometry in free equilibrium is sketched.

For the isotropic case, where $\gamma$ is a constant, the shape would be a sphere. Free surface energy minimizers are typically obtained by application of the geometrical Wulff construction [26], [9], the geometrical plot of the Cahn-Hoffmann vector, see for example [24], or numerically in terms of gradient flows that evolve with time to the equilibrium setting, for example using a level-set method [5]. However, the usual Wulff construction works only for unbounded geometries. For a nanocrystal that is attached to a solid surface, such as in solid dewetting, the geometrical idea can be extended to the Winterbottom construction [25]. Methods for calculating 
the equilibrium geometries, extending the convexification procedure of the Wulff and the Winterbottom constructions, for a range of configurations for anisotropic, even multigrain, crystals and solid surfaces have been developed recently in [27].

We also note that, based on minimization methods for anisotropic crystals in an unbounded domain, as well as attached to an isotropic surface have been discussed in [17] and [16], using software based on [4]. A different but interesting approach by [6] addresses the difficulty in obtaining accurate interfacial free energy densities directly from experimental data. A method that transforms measured equilibrated shapes in order to predict corresponding free interfacial energies is proposed and applied to various anisotropic case studies.

Our approach differs from the previously mentioned ideas, as we directly discretize both, the objective and the constraint, to formulate problem (1) as the following nonlinear, constrained, finite-dimensional minimization program

$$
\begin{array}{rl}
\min _{x \in \mathbb{R}^{N}} & f(x), \\
\text { s.t. } \quad c(x) & =0, \\
\text { for } \quad x & \geq 0 .
\end{array}
$$

Here $x$ describes the chosen parametrization of the shape that will be introduced later, $f$ is the objective, the discretized surface integral, $c$ describes the volume constraint and finally we deal with bound constraints that typically can be ignored as they are not supposed to be activated (one does not expect $x_{j}=0$ for any $j$ in the equilibrium configuration).

We will show how $(2 \mathrm{a})-(2 \mathrm{c})$ can be formulated for the unbounded problem (1). Thereafter, we extend the derivation for the more general setting, where a substrate is added to the geometrical setup. In this case the problem writes as follows,

$$
\min _{\Omega} \int_{\Gamma} \gamma d S+\int_{\Gamma_{i}} \gamma_{i} d S+\int_{\Gamma_{s}} \gamma_{s} d S \quad \text { s.t. }|\Omega|=\text { const, }
$$

so that one has to deal with three different interfaces $\Gamma_{i}, \Gamma_{s}$ and $\Gamma$. The boundary of the dot is given as $\partial \Omega=\Gamma \cup \Gamma_{i}$. Each interface brings in a corresponding interfacial energy, we write $\gamma_{i}$ and $\gamma_{s}$ for $\Gamma_{i}$ and $\Gamma_{s}$, respectively. For this setting we treat a flat substrate as it is depicted in Figure 2 (b). In the a-Si dewetting experiments this is the observed geometry, so we refrain from treating deformations at the substrate, although our approach extends generically for this setting and hence allows for more general problems.

We note that geometrical methods such as in [27] are also used in the literature. They work efficiently in case of strong anisotropies, where in equilibrium only a 
few flat facets remain. Our method uses continuum models that rely on a certain degree of smoothness and hence formulas that lead to slightly curved facets can be employed, which would lead to a demanding disposal of hyperplanes in the geometrical constructions and slows these methods down, while our minimization method works best for theses cases.

We will show that a direct minimization of problem (3), even though computationally demanding, will brings along the benefit that one can include spatial dependence in the formulas of the interfacial energies. This allows working with non-homogeneities, i.e. where the Winterbottom construction fails. In our case the surface $\Gamma$ is subdivided into different grain/orientation areas. Formally this is just a spatial dependence of $\gamma$.

The remaining parts of this work are structured as follows: First, we describe our physical problem in Section 2, where we present methods that were used to grow the nanodots of interest, discuss their interfacial energies and measured TEM pictures, which we compare with our results towards the end of our study. In Section 3 we begin with comparing the Wulff construction for well-known formulas to our results and discuss equilibrium settings for surface energy density formulations with jumps. We introduce a boundary layer formula to overcome the discontinuity problem. We show by example that the minimizers converge in the limit of a small regularization. In Section 4 we introduce the new method for equilibrium shape calculations in two dimensions. We add the substrate in Section 5 and test the method and explain the adjustments that need to be done in our minimization formulation. In Section 6 we calculate different nanodots for different substrates and surface energy densities. We carry out a parameter study concerning the positions of the grain boundaries within two-grained and three-grained nanodots. We close our work with a discussion and outlook concerning the full problem in Section 7.

\section{Synthesis of silicon nanodots}

The nanodot formation process by dewetting of a thin amorphous silicon (a-Si) layer has first been discussed by Wakayama et al. [23, 22] and has been investigated in more detail in the recent works by Malguth et al. [13] and Roczen et al. [15]. For nanodot synthesis, a thin amorphous silicon (a-Si) layer is deposited onto a $\mathrm{SiO}_{2}$ covered crystalline $\mathrm{Si}$-wafer (c-Si wafer). The a-Si/SiO $/$ c-Si system undergoes an annealing step at about $600{ }^{\circ} \mathrm{C}$ in a UHV system. This leads to the solid phase crystallization of the a-Si layer and thus to the growth of crystalline grains inside the top layer. The grains continue to grow by the consumption of the surrounding 


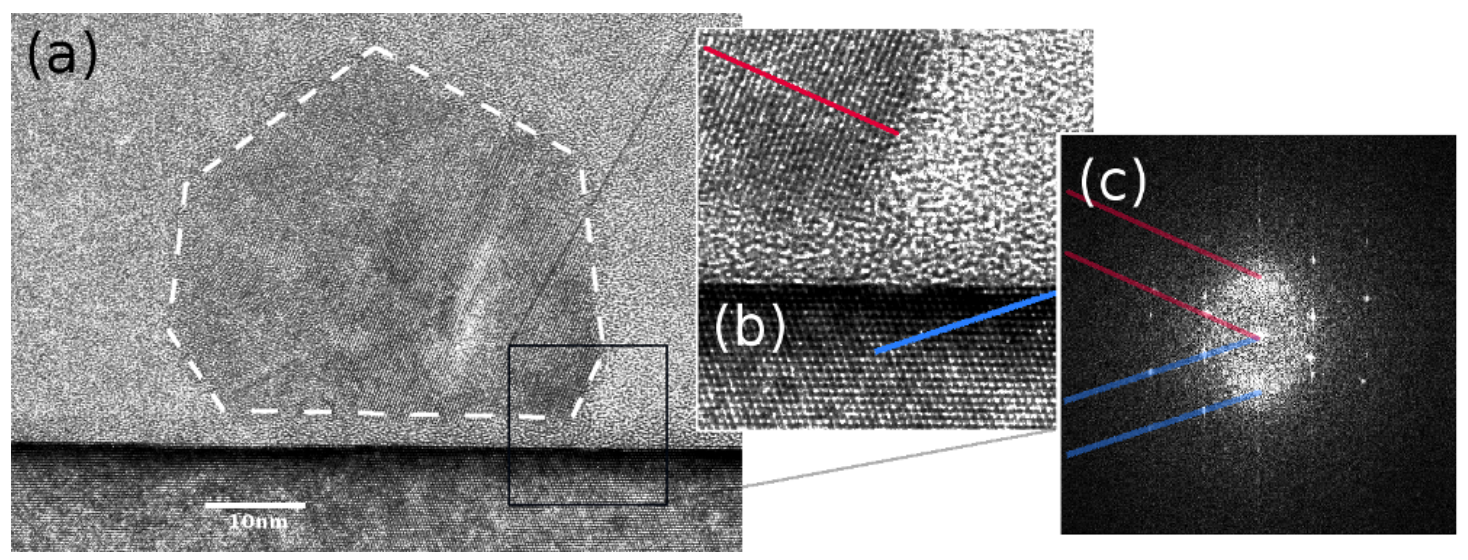

Figure 3: (a) TEM cross section image of a crystalline silicon dot sitting on a $\mathrm{SiO}_{2}$ layer that is located on top of a $\operatorname{Si}(111)$ wafer. The region to the right is magnified in (b), where characteristic orientations of the substrate and the dot are indicated by two lines; (c) shows the Fourier transform of (b) (absolute values); the noise refers to the background gel and $\mathrm{SiO}_{2}$ layer, the bright spots to the characteristic orientations of the grain and the substrate.

a-Si until the former amorphous layer is fully crystallized. Due to a surface diffusion process acting simultaneously to the recrystallization, a dewetting process takes place and an unordered array of c-Si nanodots is formed on top of the $\mathrm{SiO}_{2}$. The diameter, the density and the crystallinity of the nanodots is controlled by the thickness of the initial a-Si layer. For a sufficiently large a-Si layer thickness the nanodots become polycrystalline.

Figure 3 shows a cross sectional TEM image of a nanodot formed by the dewetting of a $20 \mathrm{~nm}$ a-Si layer. It is located on top of a $2 \mathrm{~nm}$ thick $\mathrm{SiO}_{2}$ layer and it is more than twice as high as the initial film thickness. The upper part of the nanodot is surrounded by an amorphous adhesive used for TEM sample preparation. In the lower part of the image, the single crystalline Si substrate is visible. A magnification of the lower right corner of the nanodot is depicted in (b) and its Fourier transform (the absolute values) in (c) show that the nanodot is indeed recrystallized with one orientation at this particular section of the image. While the epoxy glue and the $\mathrm{SiO}_{2}$ lead to spherically distributed noise in the Fourier transform, the $\mathrm{Si}(111)$ substrate and the dot create bright spots corresponding to their periodicity and orientation. A similar image can be obtained from Figure 1 (c), where the additional second grain adds more spots to the Fourier domain. From the transformations we cannot read off the exact three-dimensional orien- 
tations of the grains in the nanodots, however we can see that we have different crystalline, periodic structures.
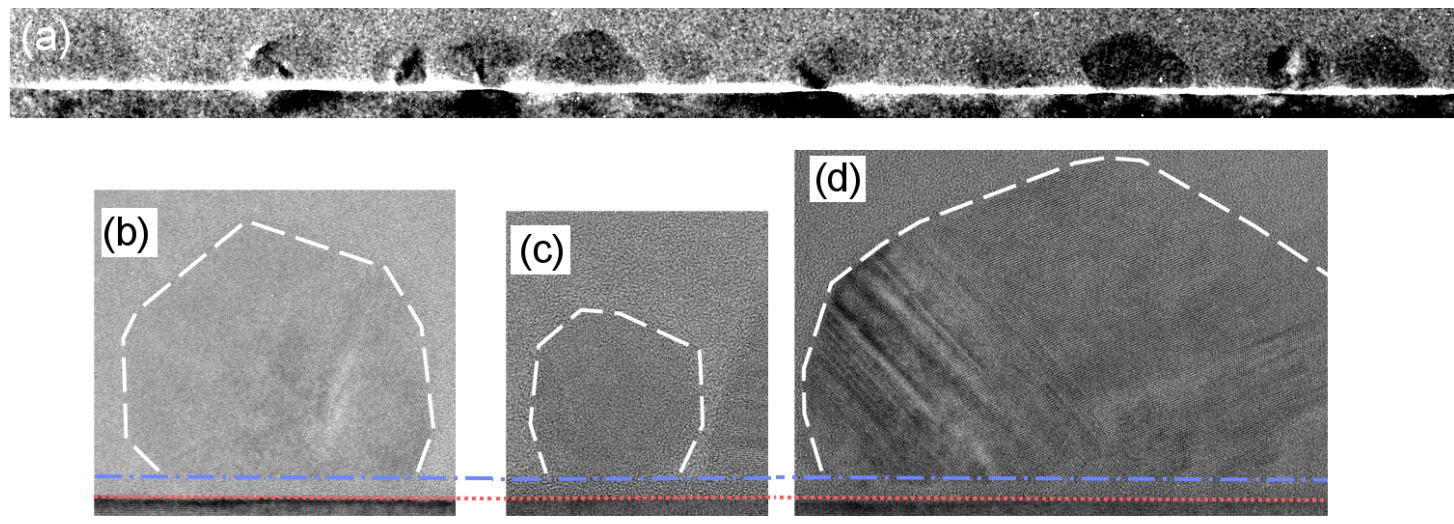

Figure 4: (a) TEM image of a dot array; initial thickness of the a-Si: $20 \mathrm{~nm}$, dewetting temperature: $700^{\circ} \mathrm{C}$. Crystalline and poly-crystalline nanodots are visible as darker shades. Better resolved nanodots are depicted in (b)-(d) in TEM images that allow for an interpretation of the different orientations.

Figure 4 shows further nanodots of the same sample. In (a) a cross sectional TEM image depicts the side profile of a nanodot-array. It is not easy to extract the exact form of the nano-islands in this resolution - this is better visible in (b)-(d), in cross sectional TEM images of single nanodots. Wakayama et al. [22] reported on a bimodal distribution of nanodots which was also observed in our experiments. Figure (c) shows one of the smaller crystals next to the wall of a bigger one and a size diagram from Figure 1 would probably show two separated maxima at two different sizes. The other TEM images in Figures 4 (b) and (d) show larger nanodots, they are poly-crystalline. We indicate their interfaces to the epoxy glue with dashed lines. One clearly sees faceting while it is difficult to detect the exact orientation of the silicon crystal grains. This is important for a detailed comparison of the experiments and calculated equilibrium shapes. However, we know now that the recrystallization interplays with a surface diffusion process that together enables the dewetting and creation of crystalline nanostructures. Furthermore, from recent c-Si dewetting experiments [1, 12], we know that the nanodots have a clearly described faceted form in the ideal one-crystalline case. For the recrystallized nanodots we need to treat orientation rotations, grain boundaries and probably other defects to fully describe nanodots in equilibrium. 


\section{$3 \quad$ Wulff shapes}

We begin with the discussion on results obtained with a well-established geometrical procedure, the Wulff construction [26]. We investigate a jump-formula and its regularization for the poly-crystalline case and describe the difficulties with this approach. Typical formulas for the surface energy density have to be extended if one plans to describe possibly poly-crystalline nanodots on $\mathrm{SiO}_{2}$ substrates. Assuming a sharp interface at the grain boundaries one has to deal with a discontinuous surface energy formula at the surface of the object of interest. Our first idea was to allow the surface energy jump at a certain orientation. This discontinuity can be regularized to form a smooth approximation. There is, however, a major problem with this approach. Due to missing orientations, the equilibrium shape has its transition from one facet to the next often at a different angle than the angle prescribed in the surface energy formula.

(a)

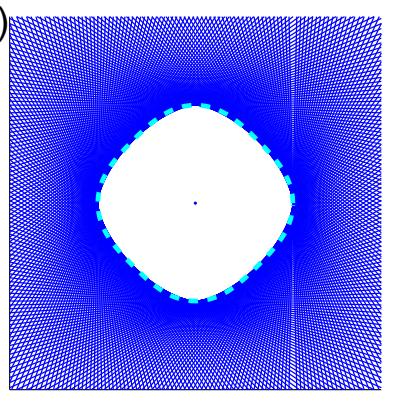

(b)

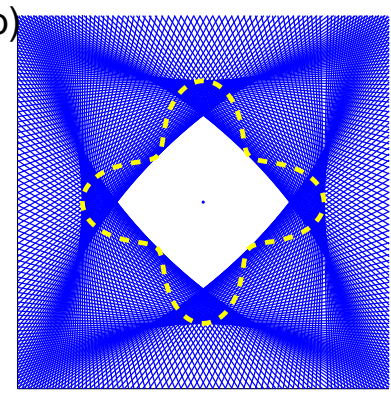

(c)

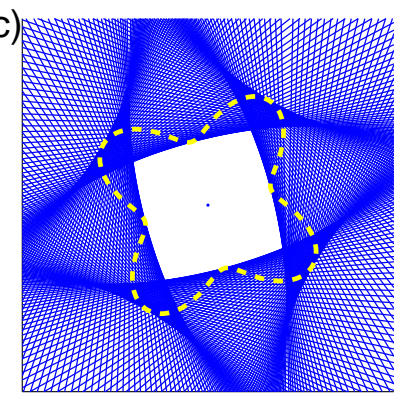

Figure 5: Polar plots (dashed lines) of the surface energy (4) with $p=4$ and the corresponding equilibrium shapes (white areas) constructed with the Wulff construction for the weak anisotropy strength $G=0.05$ in (a) and the strong anisotropy $G=0.3$ in (b); (c) shows a nonzero tilt $\varphi=\pi / 6$ in (4).

The geometrical construction for jump formulas When a formula for the surface energy density is given as function of an angle $\theta$, equilibrium shapes can be constructed geometrically. Typically, for materials with a cubic symmetry such as silicon, one can find the following $2 \mathrm{D}$ reduced, formula for the surface energy density description

$$
\gamma(\theta ; \varphi, G, p)=1+G \cos (p(\theta+\varphi))
$$

with $p=4$ (the number of minima of $\gamma$ ). Here $\varphi$ is a phase shift describing a rotation from a reference orientation. For $\varphi=0$ we calculated the equilibrium shapes 
for two different anisotropy strengths $G$ by application of the Wulff construction and plotted them in Figure 5. They turn out to be square shaped as expected and with smoothed corners in the weak case (a), $G=0.05$, or real cusps for $G=0.5$ as in (b). In (c) we observe the same shape as in (b), rotated by $\varphi=\pi / 6$.

The nanodots from the dewetting experiment as in Figure 1 have many different facets. As they may be composed of several grains that are differently oriented, the rotation parameter $\varphi$ is important and differs in the phases/facets corresponding to the individual grains. At the surface where these grains touch, a discontinuity in the surface energy density is introduced.

Our first approach for the multiple grain case is to divide the angle interval $[0,2 \pi)$ into $m$ parts, say $m=2$ and $m=3$, so that the surface can be composed of two or three portions that each have different shifts $\varphi_{k}, k=1, \ldots, m$, for now considered in the surface energy density (4). It turns out that these angles are not the angles at which the equilibrium shapes have their orientation transition. We ignore this lack of detail in this section and just assume that certain angle intervals correspond to certain prescribed orientations.

For $m=2$ we divide the angle domain into $[0, \pi) \cup[\pi, 2 \pi)$, for $m=3$ it is $[0,2 / 3 \pi) \cup[2 / 3 \pi, 4 / 3 \pi) \cup[4 / 3 \pi, 2 \pi)$ and we deal with either two or three shifts $\varphi_{k}$. We write the composed surface energy formula as

$$
\gamma^{m}(\theta)=\sum_{j=1}^{m} \mathbb{1}_{\left[\theta_{j-1}, \theta_{j}\right)} \gamma\left(\theta ; \varphi_{j}, G, p\right), \quad \theta_{j}=\frac{2 \pi}{m} j,
$$

a valid formula on the whole angle interval, using the characteristic function

$$
\mathbb{1}_{A}(x)=\left\{\begin{array}{l}
1, x \in A \\
0, x \notin A
\end{array}\right.
$$

Now we can use the composed formula (5) to carry out Wulff constructions with different strengths $G$ and with $m$ different orientations $\varphi_{j}$. However, note that the formula introduces jumps in the polar plots due to the shifts. The proof for Wulff's construction giving correct shapes relies on a surface energy density that is continuous [9]. We can calculate the equilibrium geometries as we did in Figure 6 , but we have to think about the validity of these images due to the lack of regularity. The figure shows two grain calculations in (a) and (b) for weak and strong anisotropy ( $G=0.05$ and $G=0.5$, respectively), while (c) and (d) depicts the three orientation case $m=3$ with the same values for $G$ as in (a) and (b). The dashed lines are the polar plots and it is clearly visible that they have jumps at 

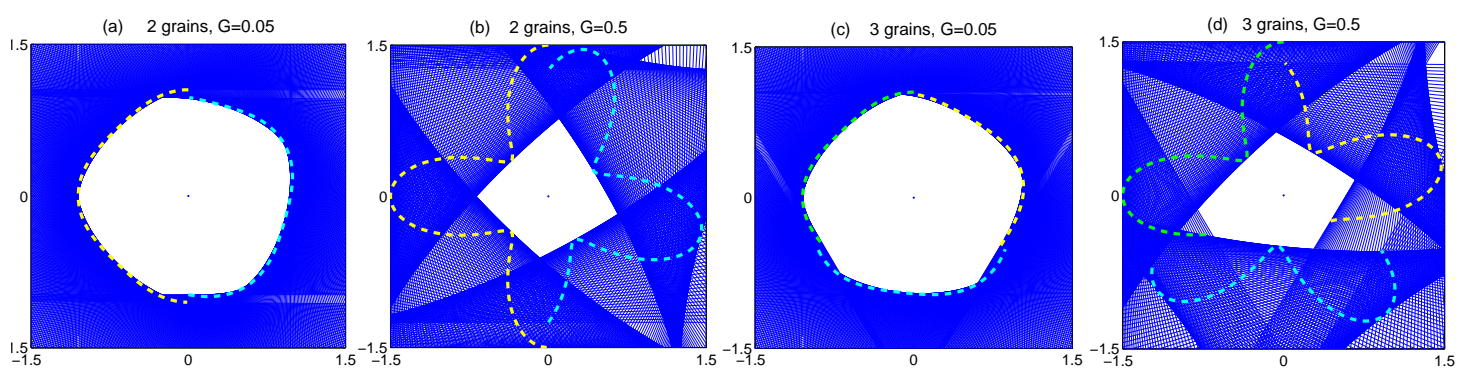

Figure 6: Wulff shapes for 2 grains in (a) and (b) with $\varphi_{1}=0, \varphi_{2}=\pi / 3$, and 3 grains, (c) and (d) with $\varphi_{1}=0, \varphi_{2}=2 / 7 \pi, \varphi_{2}=3 / 7 \pi$; the angle domains are divided in an equispaced way; (a) and (c) are calculations with a weak anisotropy $G=0.05$, while (b) and (d) show the equilibrium shapes for $G=0.5$, a strong anisotropy that induces corners. The dashed curved lines are the polar plots.

the positions, where the orientations change. The white areas are the equilibrium shapes.

To show that the Wulff shapes are indeed minimizers for the jump formula (5) we introduce a boundary layer formula that smoothes out the jumps in the surface energy density on a characteristic small length-scale $\epsilon$. As this formula is continuous its Wulff shapes are correct minimizers. It is an extension of the boundary layer model discussed before by several authors [11, 19] and applied successfully for dynamical models describing the self-assembly of heteroepitaxially grown quantum dot arrays $[20,10]$. Thus we shortly write $\gamma_{j}=\gamma\left(\theta ; \varphi_{j}, G, p\right)$ and define

$$
\gamma_{b l, j}(\theta ; \epsilon)=\frac{1}{2}\left(\gamma_{j+1}+\gamma_{j}\right)+\left(\gamma_{j+1}-\gamma_{j}\right) \frac{1}{\pi} \arctan \left(\frac{\theta-\theta_{j}}{\epsilon}\right) .
$$

It has to be assumed that $\epsilon \ll \min _{j}\left\{\left|\theta_{j+1}-\theta_{j}\right|\right\}$, then the formula

$$
\gamma_{b l}^{m}(\theta)=\sum_{j=1}^{m} \mathbb{1}_{\left[\theta_{j}-\Delta \theta_{j}, \theta_{j+1}-\Delta \theta_{j+1}\right)} \gamma_{b l, j}, \quad \Delta \theta_{j}=\frac{\theta_{j}+\theta_{j-1}}{2}
$$

is indeed a smoothed regularization. There is still a possible discontinuity at the new angle boundaries $\theta_{j}-\Delta \theta_{j}$, the former medians of the angle intervals. However, the tails of the neighboring arctan function converge exponentially fast to the same constant far-field values, so that we ignore this lack of regularity, which vanishes in the $\epsilon \rightarrow 0$ limit.

Figure 7 (a) depicts how a sequence of decreasing values for $\epsilon$ influences the polar plot of $\gamma_{b l}^{m}$. The discontinuities are approximated more precisely with smaller $\epsilon$. The shapes in (b) show that even for the larger regularization coefficient $\epsilon=0.1$ 
the equilibrium shape nearly matches that for the discontinuous $\gamma^{m}$. For very small $\epsilon$ the difference is marginal. Note that here we have a rather extreme case, where the discontinuities are large and we deal with a nonconvex surface energy density due to a large anisotropy coefficient $G$. We conclude that the approximation (6) works well and that the jumps in (5) are not a major problem. However, Figure 7 (c) explains why our idea fails. While the angle at the jump is supposed to describe the location of the material change, it does not in general. We will show a better way to treat the interface position in the following sections.

(a)

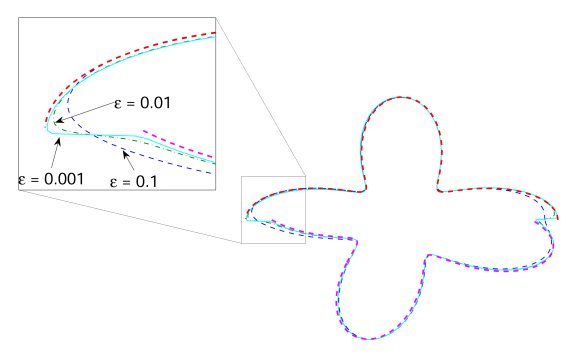

(b)

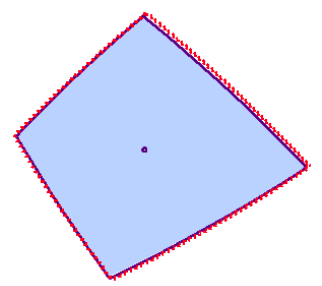

(c)

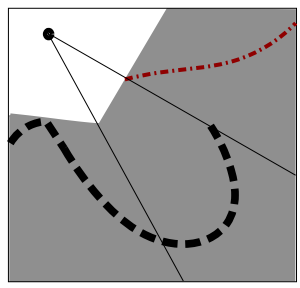

Figure 7: Polar plots for $\gamma^{m}$ and $\gamma_{b l}^{m}$ with $m=2$, anisotropy strength $G=0.5$ from (5) and (6) with $\epsilon=0.1,0.01,0.001$ in (a). The left discontinuity at $\theta=\pi$ is magnified and one sees the smoothing effect of the boundary layer formula. The shifts are $\varphi_{1}=0$ in $[0, \pi)$ and $\varphi_{2}=\pi / 3$ in $[\pi, 2 \pi)$; The image in (b) shows the equilibrium shape for the non-continuous version (filled area) and the shapes for $\epsilon=0.1$ (dashed line) and $\epsilon=0.001$ (solid line); (c) sketch for the difference between the jump angle in the surface energy and in the angle of the parametrization of the actual equilibrium shape

So far we have not considered the interaction with a substrate and this can affect the equilibrium shapes significantly. In the next Section we proceed with the numerical discretization method for the unbounded case first, then, in Section 5 the method is extended to the substrate case. There we show how we deal with orientation transitions at the surface for a fixed parametrization angle. We are not aware of a Wulff construction based method that could resolve the position of the interface as in our method. 


\section{Numerical optimization formulation for the un- bounded case}

For our numerical method we derive the discretized minimization formulation that transforms the original problem (1) to the mathematical form (2a)-(2c). We first assume that the equilibrium shape is star-shaped. We assume that the equilibrium shape is star-shaped and show how the continuous domain shape will be related to the finite-dimensional variable $\mathrm{x}$ in this formulation. Let us note that the optimization routine will change the discretized $\Gamma$ in each iteration. Therefore we introduce the following parametrization of the shape in the $j$ th iterate during optimization

$$
\Gamma:[0,2 \pi) \times \mathbb{N} \rightarrow \mathbb{R}^{2}, \quad(\theta, j) \mapsto\left(\Gamma_{1}(\theta, j), \Gamma_{2}(\theta, j)\right) .
$$

For the outward unit normal of the sphere we write $n=n(\theta)$, the tangent is defined by $t=t(\theta), \theta \in[0,2 \pi)$ and to indicate the actual shape in the $j$ th iterate $\Gamma(\theta, j)$ we use $\Gamma$ superscripts, $n^{\Gamma}=n^{\Gamma}(\Gamma(\theta, j)), t^{\Gamma}=t^{\Gamma}(\Gamma(\theta, j))$ (see also Figure 8).

Problem (1) requires to treat the free energy and the mass constraint that both can be expressed in terms of integrals. Parametrization (7) changes these integrals in the following way, the mass constraint reads

$$
M=\int_{0}^{2 \pi} \int_{0}^{|\Gamma(\theta, j)|} \rho d \rho d \theta=\int_{0}^{2 \pi} \frac{1}{2}|\Gamma(\theta, j)|^{2} d \theta
$$

and the objective $J(\Omega)=\int_{\partial \Omega} \gamma d S$ becomes

$$
J(\Gamma)=\int_{\Gamma} \gamma(\Theta) d s=\int_{0}^{2 \pi} \gamma(\Theta(\Gamma(\theta, j)))\left|\partial_{\theta} \Gamma(\theta, j)\right| d \theta
$$

Note that $\partial_{\theta} \Gamma=t^{\Gamma}$. We use the initial condition $\Gamma(0)=\Gamma_{0}=\left\{x \in \mathbb{R}^{2}:|x|=1\right\}$ that can be discretized equidistantly on the grid $0=\theta_{0}<\theta_{1}<\ldots<\theta_{N}<2 \pi$, with $\theta_{k}=k d \theta, k=0,1, \ldots, N, d \theta=2 \pi /(N+1)$ via

$$
\theta_{k} \mapsto\left(\Gamma_{0}\right)_{k}=\left(\cos \left(\theta_{k}\right), \sin \left(\theta_{k}\right)\right)
$$

and one reads off the normals and tangents

$$
n_{k}=n\left(\theta_{k}\right)=\left(\Gamma_{0}\right)_{k}, \quad t_{k}=t\left(\theta_{k}\right)=\left(\partial_{\theta} \Gamma_{0}\right)_{k}=\left(-\sin \left(\theta_{k}\right), \cos \left(\theta_{k}\right)\right) .
$$

The shape described by $\Gamma$ is related to the discretization angles $\theta$. However, the objective, i.e. the surface energy $\gamma$, will depend on angles that $\Gamma$ forms to some reference orientation $e_{r e f}$, which differ from $\theta$, we denote them by $\Theta$. More precisely, 
these are the angles the tangents at the surface form with $e_{r e f}$. In the discretized case we write $\gamma_{k}=\gamma\left(\Theta_{k}\right)$, where the $\Theta_{k}$ are the angles determined by the actual discrete geometry of the $2 \mathrm{D}$ crystal, i.e. neighboring points. Therefore, we consider

$$
a_{k}=\left(\left(a_{k}\right)_{1},\left(a_{k}\right)_{2}\right)=\left(\left(\Gamma_{k+1}\right)_{1},\left(\Gamma_{k+1}\right)_{2}\right), \quad b_{k}=\left(\left(b_{k}\right)_{1},\left(b_{k}\right)_{2}\right)=\left(\left(\Gamma_{k}\right)_{1},\left(\Gamma_{k}\right)_{2}\right)
$$

with $\Gamma_{k}=\Gamma\left(\theta_{k}, j\right)$ and we define $w_{k}=a_{k}-b_{k}$, where we use periodicity at the boundaries of definition. We calculate the angle that the vector $w_{k}$ forms with the first Cartesian basis element $(0,1)$. Doing so for the whole shape, we get a vector of angles,

$$
\Theta_{k}=\arccos \left(w_{k} \cdot(0,1)^{T} /\left|w_{k}\right|\right), \quad k=1, \ldots, N .
$$

We could work with a discretized shape $\Gamma$ that is free to be changed in whole $\mathbb{R}^{2}$ by the optimization procedure. However, this leads to certain problems, i.e. the nodes can separate strongly and destroy the anticipated accuracy expected by the theory. Hence we proceed in the following much more efficient way: Instead of optimizing for $\left(\left(\Gamma_{k}\right)_{1},\left(\Gamma_{k}\right)_{2}\right)_{k=1, \ldots, N}$ in $\mathbb{R}^{2}$, we reduce one free parameter by allowing change only along beams outgoing from the origin. Therefore we rewrite all quantities by introducing polar coordinates.

The surface is interpreted as a mapping from the sphere (and initial condition) $\Gamma_{0}$,

$$
\Gamma(\theta, j)=x(\theta, j) \Gamma_{0}(\theta)=x n
$$

and we optimize for $x$ instead of $\Gamma$. The derivative becomes

$$
\partial_{\theta} \Gamma(\theta, j)=t^{\Gamma}=x^{\prime} n+x t
$$

and its 2-norm becomes due to the orthogonality property of normal and tangent of the sphere

$$
\left|\partial_{\theta} \Gamma(\theta, j)\right|=\left(x^{2}+\left(x^{\prime}\right)^{2}\right)^{1 / 2} .
$$

The integrals are transformed in the following way

$$
\int_{\Gamma} \gamma d S=\int_{0}^{2 \pi} \gamma(\Theta(x)) \sqrt{x^{2}+\left(x^{\prime}\right)^{2}} d \theta, \quad \int_{\Omega} d V=\int_{0}^{2 \pi} \frac{1}{2} x^{2} d \theta .
$$

The angle the surface forms at the position $\theta$ with some fixed orientation is $\Theta(x)=$ $\Theta(x(\theta))$, and it can still be calculated as before. Now we have an expression for the two quantities in terms of the radial coordinate $x$. When we discretize $\theta$ equidistantly, we march along the equally distributed normals of a circle until crossing $\Gamma$ to get the approximations $\Gamma_{j}$. This is sketched in Figure 8. The iterates 


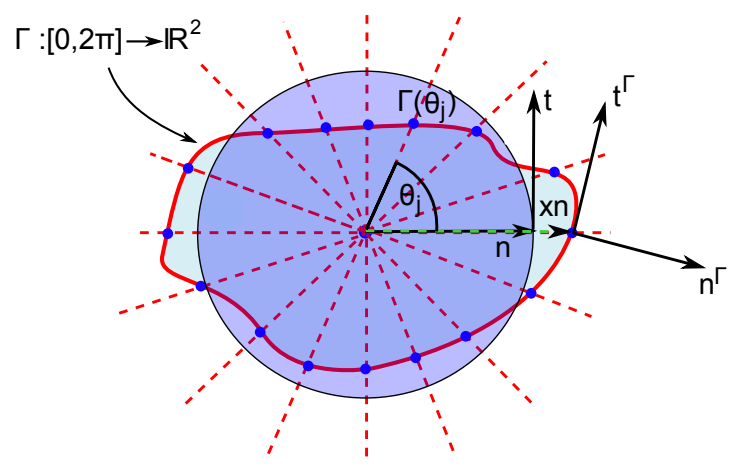

Figure 8: The thick red line is given by the parametrization $\Gamma$. For the discretization we intersect $\Gamma$ with equidistantly distributed normals of the sphere.

are bound to those directions prescribed by the normals, i.e. the discretized angle domain.

The discretization gives in each iteration the values $\left(x_{k}\right)_{k}=\left(x\left(\theta_{k}\right)\right)_{k}$. We approximate the integrals in (13) with an appropriate integration rule that relies on these values. We apply Simpson's rule that gives for a function $f$ on a $[0,2 \pi)$ interval

$$
\int_{0}^{2 \pi} f d \theta \approx \frac{d \theta}{3}\left[\frac{f\left(\theta_{0}\right)+f\left(\theta_{N}\right)}{2}+\sum_{k=1}^{N-1} f\left(\theta_{k}\right)+2 \sum_{k=0}^{N-1} f\left(\frac{\left(\theta_{k+1}+\theta_{k}\right)}{2}\right)\right] .
$$

We use it with $f(\theta)=\gamma(\Theta(x)) \sqrt{x^{2}+\left(x^{\prime}\right)^{2}}$ and $f(\theta)=\frac{1}{2} x^{2}$.

For the discretization $x=\left(x_{k}\right)_{k=0,1, \ldots, N} \geq 0$ we can use any finite difference matrix $D$ to obtain the discretized derivative $D x$. We use a simple forward difference, as central differences can lead to zig-zagging effects here.

Summarizing, we can formulate the nonlinear equality constrained optimization problem with bound constraints on the variables as written down in (2a)-(2c). The initial guess is $x_{j}=1, j=0,1, \ldots, N$, which is feasible by construction when the volume constraint is set to that of the unit ball, $M=\pi$, else one needs to scale the $x_{j}$ accordingly. Using MATLAB's fmincon routine (from the Optimization Toolbox) one finds, that the positivity inequalities may be ignored in the cases presented here. As expected the final, but also the intermediate shapes, do not violate the inequality constraints, so that it may be sufficient to practically work with (2a) and (2b) and ignore (2c).

Comparisons to Wulff shapes Now we have two methods at hand that both lead to equilibrium shapes of anisotropic crystals. 
(a)

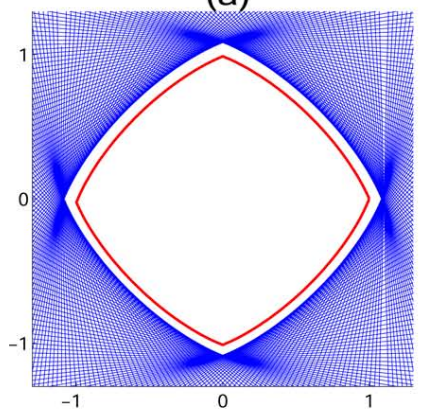

(b)

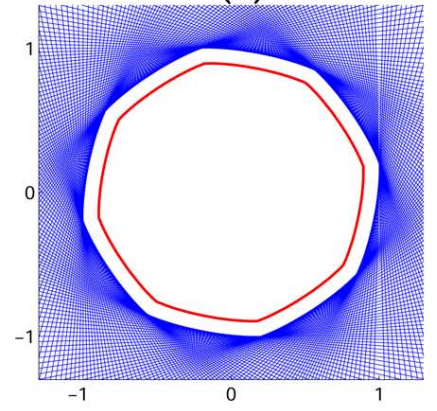

(c)

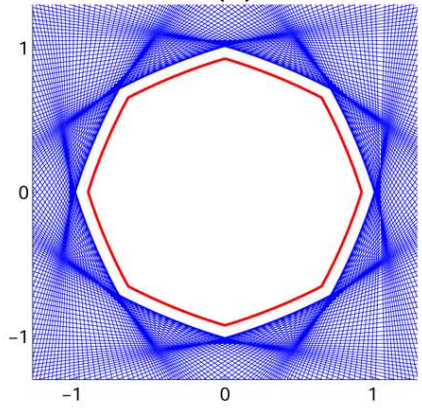

Figure 9: Comparison between Wulff shapes (white areas) and solutions with the minimization procedure (inside solid curve) calculated with $N=256$ grid points. Formula (4) with (a) $(p, G, \varphi)=(4,0.1,0)$; (b) $(p, G, \varphi)=(8,0.02,-\pi / 16)$; (c) $(p, G, \varphi)=(8,0.05,0)$

Figure 9 shows Wulff constructions for various formulas of the surface energy density. The solid lines depict the equilibrium shapes calculated with the numerical method described above. The shapes in (a)-(c) are exactly the same. We have chosen a volume in the minimization procedure that is slightly smaller than that of the Wulff shape to be able to see the solid line well on the white shape. We applied our procedure to formula (4) with different values of $p, G$ and $\varphi$. In (a) the standard values $(p, G, \varphi)=(4,0.1,0)$ have been chosen and show the squareshaped equilibrium; In (b) and in (c) we used an 8-fold symmetry, $p=8$; in (b) a rotation of $\varphi=-\pi / 16$ is well captured and in (c) (here $\varphi=0$ ) the increase of the anisotropy strength from $G=0.02$ to $G=0.05$ is shown.

\section{Numerical optimization for nanodots on a rigid substrate}

We now take into account an adhesive force due to the contact of the nanodots with the substrate. We work with the energies between the vacuum phase (or gas) and the nanodot, $\gamma$ acting on $\Gamma$, the substrate and the nanodot, $\gamma_{i}$ belonging to $\Gamma_{i}$, and the substrate and the vacuum, $\gamma_{s}$ related to $\Gamma_{s}$. As depicted in Figure 2 (b) we consider the case where $\Gamma_{s}$ and $\Gamma_{i}$ are flat. For comparison with experimental measurements, as depicted in Figure 1, 3, 4, we need to extend the minimization problem (1) and treat (3) in this Section. We will show how to adjust the previously introduced optimization procedure to solve the problem. As the substrate is rigid, 
the two additional integrals in (3) become very simple. We can rewrite the problem in this case as

$$
\min _{\Omega} \int_{\Gamma} \gamma d S+x_{r}\left(\gamma_{i}-\gamma_{s}\right)+x_{l}\left(\gamma_{s}-\gamma_{i}\right) \quad \text { s.t. }|\Omega|=\text { const, }
$$

where $x_{r}$ is the $x$-coordinate of the right triple point and $x_{l}$ is the corresponding left value. The same reformulation has been used to derive the Winterbottom construction [25]. We assume that $x_{l} \leq 0$ and $x_{r} \geq 0$. As sketched in Figure 10, we can discretize similarly as before for $\Gamma$, only that now we use only half the sphere, i.e. angles in $[0, \pi]$, and we lose periodicity.

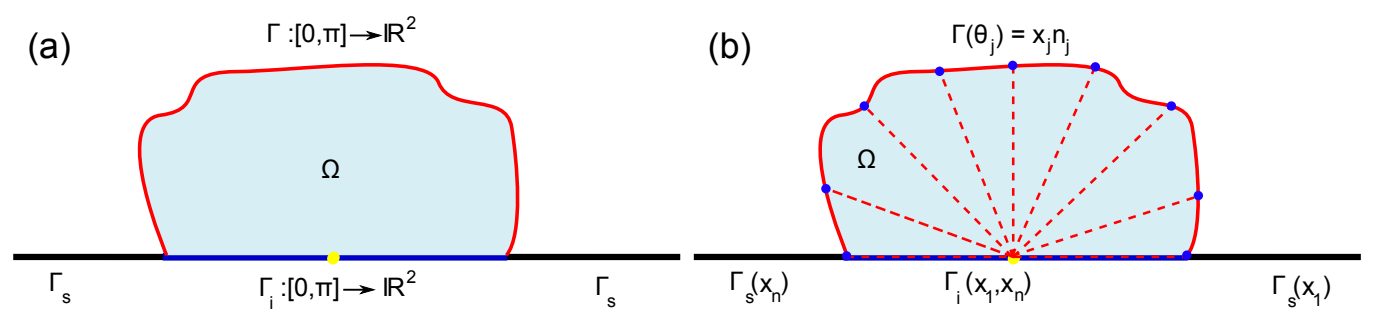

Figure 10: (a) All three interfaces, $\Gamma, \Gamma_{i}, \Gamma_{s}$ are mappings from $[0, \pi]$ to $\mathbb{R}^{2}$; (b) shows the discretization for all three interfaces in terms of polar coordinates.

The parametrization of the surface $\Gamma$ is as in (7), restricted to the domain $[0, \pi]$, with the volume and objectives, i.e. in (8) and (9), adjusted to the shorter interval $[0, \pi]$.

We work with similar expressions as in (13) and solve

$$
\min _{x \geq 0} f(x)=\int_{0}^{\pi} \gamma(\Theta(x)) \sqrt{x^{2}+\left(x^{\prime}\right)^{2}} d \theta+x_{r}\left(\gamma_{i}-\gamma_{s}\right)+x_{l}\left(\gamma_{s}-\gamma_{i}\right)
$$

subject to the mass constraint

$$
c(x)=\frac{1}{2} \int_{0}^{\pi} x^{2} d \theta-M=0
$$

and the bound constraint $x \geq 0$. Note that we can use a spatial dependence of $\gamma$ by adding an additional separate dependence on the parametrization angle $\gamma=\gamma(\Theta, \theta)$, and hence we are able to use different orientations of one basis formula at different intervals of $\theta$.

Aspects of the discretization To be able to calculate equilibrium shapes that adhere to a substrate as sketched in Figure 2 (b), we have formulated the minimization procedure in terms of $\Gamma, \Gamma_{i}, \Gamma_{s}, \gamma, \gamma_{i}$ and $\gamma_{s}$ and simplified it by using 
polar coordinates to get problem (16) with the constraint (17). The integrals at the rigid interfaces $\Gamma_{s}$ and $\Gamma_{i}$ were further simplified to tractable algebraic expressions. Note that $x_{l}$ and $x_{r}$ in the rewritten objective (16) are the boundary points of the unknown vector $x$, i.e. $x_{\mid \theta=0}=x_{r}$ and $x_{\mid \theta=\pi}=-x_{l}$.

When we apply Simpson's rule to (14), we need derivative approximations for $x^{\prime}$ and the angles $\Theta$ for the surface energy density evaluation. We proceed in the following way: With an equidistant discretization of the angle domain,

$$
\left\{\theta_{j}\right\}_{j=0,1, \ldots, N}, \quad \theta_{j}=\frac{\pi}{N} j, \quad\left\{x_{j}\right\}_{j=0,1, \ldots, N}, \quad x_{j}=x\left(\theta_{j}\right),
$$

we have a problem defining the same number of forward derivatives. Therefore we work with averaged quantities $\left\{\left(\bar{\theta}_{j}, \bar{x}_{j}\right)\right\}_{j=1, \ldots, N}$, here the arithmetical mean over neighboring points. We define the corresponding $N$ derivatives $\left\{\bar{x}_{j}^{\prime}\right\}_{j=1, \ldots, N}$ just as the finite differences $\bar{x}_{j}^{\prime}=\left(x_{j}-x_{j-1}\right) / d \theta$ and finally we approximate $x_{r} \approx \bar{x}_{1}$ and $x_{l} \approx \bar{x}_{N}$. Note that the angles $\Theta_{j}$ are calculated as in (12), with the caveat, that the vectors in (11) are now defined by the averaged variables $\left\{\bar{x}_{j}\right\}_{j}$. The main integral in the objective is now interpreted in terms of the bar variables and again it can be evaluated with Simpson's approximation (14).

For the initial condition we use a half-sphere

$$
\Gamma_{0}(M)=\left\{r(\cos (\theta), \sin (\theta)): \theta \in[0, \pi], \int_{-r}^{r} r \sin (\theta) d \theta=M\right\},
$$

which we can do because the initial contact angles do not need to be accurate in this method. For given volume, the integral constraint directly gives $r=\sqrt{2 M / \pi}$ and for the discretized case we use an initial vector $x_{j}=r$ for all components. For the parameter studies that will follow in Section 6 we always used the gained solution of the last parameter set as initial condition, when continuing for different parameters close-by.

Comparisons of the results of our new optimization method with results of the well-understood isotropic case, where equilibrium contact angles are known when the constant energy densities $\gamma, \gamma_{i}$ and $\gamma_{s}$ are prescribed (see e.g. [7]), showed good agreement. We obtain the hemispherical shapes as expected, with correct contact angles and the observed quantities converge to the expected limit. We next extend our method to the anisotropic case.

The anisotropic case - academic, single grained Figure 11 shows our results for surface energy density $\gamma$ defined by formula (4), where we increase $\gamma_{i}$ from 0.5 to 1.5 , while keeping $\gamma_{s}$ constant. Note that there are more choices to make in 

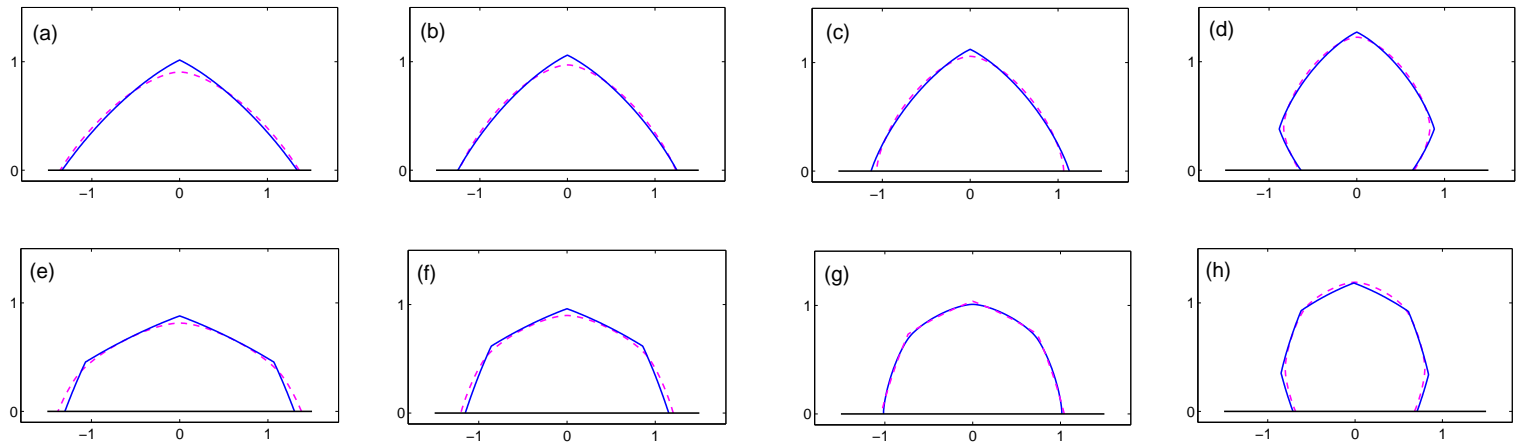

Figure 11: Equilibrium structures for $\gamma=1+G \cos (p \theta)$ for $\gamma_{s}=1$ and three different values of $\gamma_{i}$; top row: $p=4$ with $G=0.05$ (dashed line) and $G=0.1$ (solid line); bottom row: $p=8$ with $G=0.01$ (dashed line) and $G=0.05$ (solid line); (a), (e) $\gamma_{i}=0.5$; (b), (f) $\gamma_{i}=0.7$; (c), (g) $\gamma_{i}=1 ;$ (d), (h) $\gamma_{i}=1.5$.

formula (4). In the upper row of the figure, we have chosen $p=4$, while the lower row is calculated for $p=8$. Each subplot shows two equilibria due to different anisotropy strength, $G=0.05, G=0.1$ in (a)-(d) and $G=0.01, G=0.05$ in (e)(h). Similarly as in liquid droplets, as the interface energy density $\gamma_{i}$ grows, the creation of the $\Gamma_{i}$ interface is less preferred, the structures are squeezed to shorten this interface. An additional effect due to the anisotropy is visible in (d) and (h), where the adhesive equilibrium structures create additional facets to minimize the overall surface free energy. Note that the facets get more pronounced for the same values of $G$, when $p$ is increased. Sharp facets are introduced when $\gamma+\gamma^{\prime \prime}<0$. As $p$ grows, a larger quantity is subtracted from $\gamma$ explaining this observation and the stronger influence of the anisotropy parameter $G$.

The anisotropic case - academic, multiple grains We can proceed as in the unbounded case and calculate equilibria with several orientational shifts. In each of the angle sections within the $[0, \pi]$ domain we prescribe a different orientation $\varphi$ in formula (4). Figure 12 shows the result for $p=4$ and $G=0.1$. In all three subfigures we see three islands, as we varied the interfacial energy as before, here $\gamma_{i}$ takes on the values $0.5,1$ and 1.5. While increasing the values, the structures reduce the length of their base and grow vertically. In (a) only a single orientation as before was used, but here $\varphi=\pi / 3$, introducing already some asymmetry. In (b) and (c) we added one, respectively two, orientations, $\varphi=0, \varphi=\pi / 6$. We divided the angle interval $[0, \pi]$ into two and three, respectively, equally large sections of 
the parametrization domain for which we used the different shifts. Note, that in this way the angular domain is equispaced but not the surface areas. Hence the surface areas of the islands with varying orientations may be different in these figures. To obtain an equilibrium that allows the interface of neighboring grains to be free, one can iterate through all orientations as we do in the next section for a more realistic formula for $\gamma$.
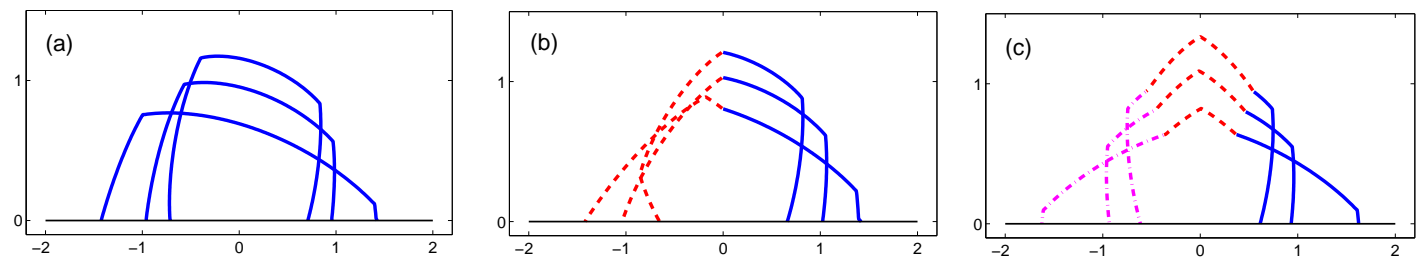

Figure 12: Equilibria for $\gamma=1+G \cos (4 \theta+\varphi)$ for $\gamma_{s}=1$, in each plot with three different values of $\gamma_{i}$ and anisotropy strength $G=0.1$; discretization with $n=257$ points; (a) one orientation, $\varphi=\pi / 3$; (b) two orientations, $\varphi=\pi / 3$ for $\theta \in[0, \pi / 2$ ) and $\varphi=0$ for $\theta \in[\pi / 2, \pi]$; (c) three orientations, $\varphi=\pi / 3$ for $\theta \in[0, \pi / 3), \varphi=0$ for $\theta \in[\pi / 3,2 \pi / 3)$ and $\varphi=\pi / 6$ for $\theta \in[2 \pi / 3, \pi]$.

\section{Experimental shapes and comparisons}

We now return to the experimental results involving poly-crystalline silicon nanodots. The surface energy density derived from experimental measurements from a void in a particular plane of silicon is plotted in Figure 13 (a), where we see the normalized energy $\gamma / \gamma(111)$ with $\gamma(111)=1.23 \cdot 10^{-4} \mathrm{~J} / \mathrm{cm}^{2}$, see [8]. The extrema are actually quite different from each other in shape and in magnitude, hence the previously used formula (4) differs qualitatively from the realistic setting, no matter which values of $G, p$ or $\varphi$ are chosen.

The polar plot and the corresponding Wulff shape are depicted in (b), which is shown again together with a TEM image taken from Eaglesham et al. [8] in (c). It shows that the trigonometric interpolant which we have implemented indeed defines the equilibrium void geometry, which has to be expected as the original formula has been extracted from this geometry. To set up the interpolant, $N$ discrete values $u_{k}=\gamma\left(\theta_{k}\right)$ have been read off from the original work, these allow to define the trigonometric function

$$
I_{N} \gamma(\theta)=\sum_{k \in \mathcal{K}} \hat{u}_{k} e^{i k \theta}
$$


where the coefficients $\hat{u}_{k}$ are discrete Fourier coefficients and $\mathcal{K}$ is a suitable set of wavenumbers. This interpolant enables an evaluation of the surface energy density formula approximation for arbitrary $\theta$. The equilibrium shape corresponding to this formula differs from that relying on the typically used cosine model with $p=4$. We obtain multiple facets that are rather rounded than pronounced planar parts. Two grain calculations can now be carried out by employing the physically derived
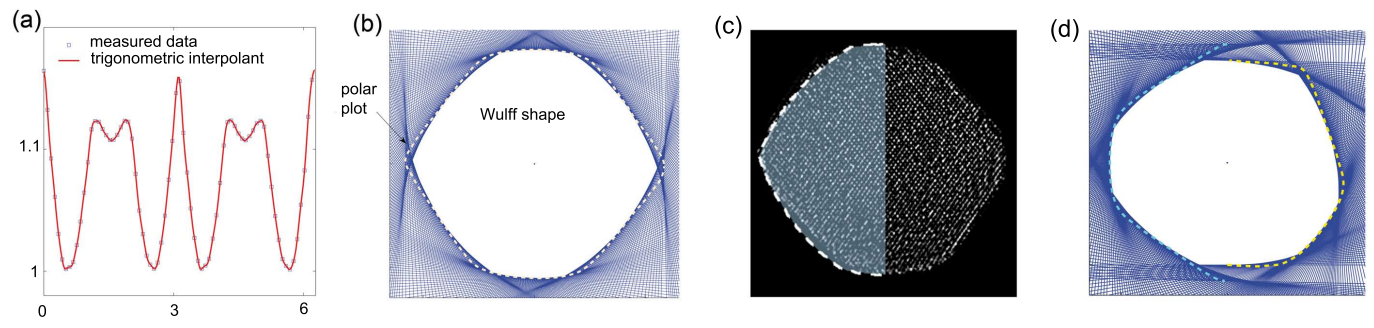

Figure 13: (a) Surface energy density of silicon, $\gamma(\theta) / \gamma(111)$; discrete values read off from Eaglesham et al. [8], who determined $\gamma(111)=1.23 \mathrm{~J} / \mathrm{m}^{2}$; solid line: approximation of the surface energy density of silicon for all angles via trigonometric interpolation; (b) the corresponding polar plot and Wulff plot for the equilibrium shape; (c) Equilibrium shape by Eaglesham et al. (adaptation) and one half of the Wulff shape, calculated by using the trigonometric interpolant; (d) Polar plot and equilibrium shape for two rotated grains ( $\pi / 5$ misorientation) with trigonometric interpolation of measured data.

formula, see Figure $13(\mathrm{~d})$. Thus, we use $I_{n} \gamma(\theta)$ for the angles in the range $\theta \in[0, \pi)$ and $I_{n} \gamma\left(\theta+\theta_{\text {rot }}\right)$ in $[\pi, 2 \pi)$, where $\theta_{\text {rot }}=\pi / 5$ is the rotation of the second grain. In comparison to the one-orientation case the symmetry is lost and indeed suggests that the asymmetric nanodots from the experiments can result from the different orientations of neighboring grains.

To add a substrate we need to quantify values for $\gamma_{i}$ and $\gamma_{s}$, the interfacial energies between $\mathrm{SiO}_{2}$ and crystalline silicon, and between $\mathrm{SiO}_{2}$ and vacuum. For the interface energy between crystalline silicon and the $\mathrm{SiO}_{2}$ a much smaller value is given in [18], $\gamma_{i}=5 \cdot 10^{-6} \mathrm{~J} / \mathrm{cm}^{2}$. The interfacial energy of $\mathrm{SiO}_{2}$ to the vacuum has been cited in [14] to be between 40 and $50 \mathrm{mN} / \mathrm{m}$, which corresponds to $\gamma_{s}=4 \cdot 10^{-6} \mathrm{~J} / \mathrm{cm}^{2}-5 \cdot 10^{-6} \mathrm{~J} / \mathrm{cm}^{2}$, where we used $\gamma_{s}=4 \cdot 10^{-6} \mathrm{~J} / \mathrm{cm}^{2}$ for all of our subsequent simulations. After normalization with $\gamma(111)$ we take $\gamma_{i} \leftarrow$ $\gamma_{i} / \gamma(111)=0.0407$ and $\gamma_{s} \leftarrow \gamma_{s} / \gamma(111)=0.0325$

To fix the interface position in a poly-crystalline nanodot, we prescribe the corresponding angle in the parametrization. We then iterate through all possible 
interface positions, calculate the corresponding energy value and pick the smallest one to indeed have the most preferred equilibrium shape. The nanodots under consideration are relatively big and additional effects that are not considered here can fix the interface to a position that does not correspond to the position of the global surface energy minimum over all interface position angles. It is quite common that while during recrystallization the interfaces move fast, they stagnate once they meet, or at least move on a very slow time scale. These states, with rather stable interface positions, are then of practical interest.
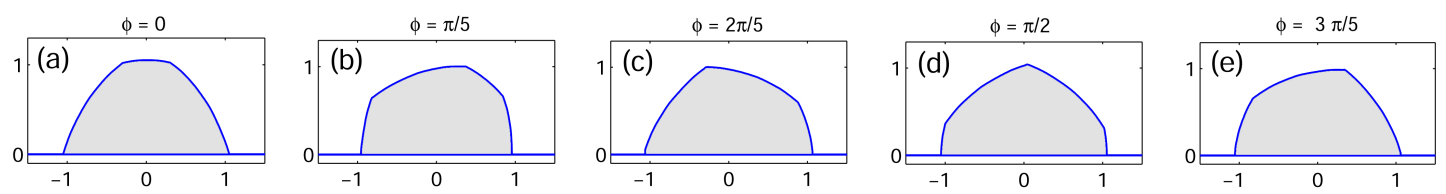

Figure 14: Five nanodots calculated with Eaglesham's formula and the rotations $\phi=\theta_{\text {rot }}=0, \pi / 5,2 \pi / 5, \pi / 2,3 \pi / 5$, successively in (a)-(e).

We first consider a single crystal nanodot located on the $\mathrm{SiO}_{2}$. To generate Figure 14, a range of orientations for the crystalline phase has been tested. All the nanodots depicted in (a)-(e) show states with different rotation from the $\mathrm{Si}(111)$ formula $\phi=\theta_{\text {rot }}=0, \pi / 5,2 \pi / 5, \pi / 2,3 \pi / 5$. The symmetry from the plot in Figure 13 (a) is visible in Figure 14 (c) and (e) as the surface energy density is locally symmetric around the state in (d) - i.e. the $\{110\}$ mirror plane - and $2 \pi / 5$ and $3 \pi / 5$ are both $\pi / 10$ from $\pi / 2$ away. We are not in the position to make one-to-one comparisons between the calculated shapes and e.g. TEM images of nanodots, as these do not give the exact orientation of the crystal and because we do not have formulas of the surface energy density for all planes of silicon at hand. However, the images show that the used formula and the parameters work well together and that the contact angles are close to those observed in experiments - and so is the asymmetric multi-faceted appearance. Also, the typical slopes of the nanodots at the substrate are large. However, let us mention that the new works [1, 12] obtained one-crystalline nanodots that exhibit more facets, hence additional minima in the formula of the surface energy density. The formula we use might still not be precise enough. We will investigate this further in future.

Next, we applied the method for two-grained nanodots. For the results in Figure 15 we fixed the orientation of the two grains, $\theta_{\text {rot }}=\pi / 3$ and $\theta_{\text {rot }}=\pi / 5$, and iterated over all angles $\theta \in[0, \pi]$ at which the interface between the two phases can be located. For each we solved the minimization problem and calculated the value of the objective of the minimizer constrained to this particular angle. In Figure 15 

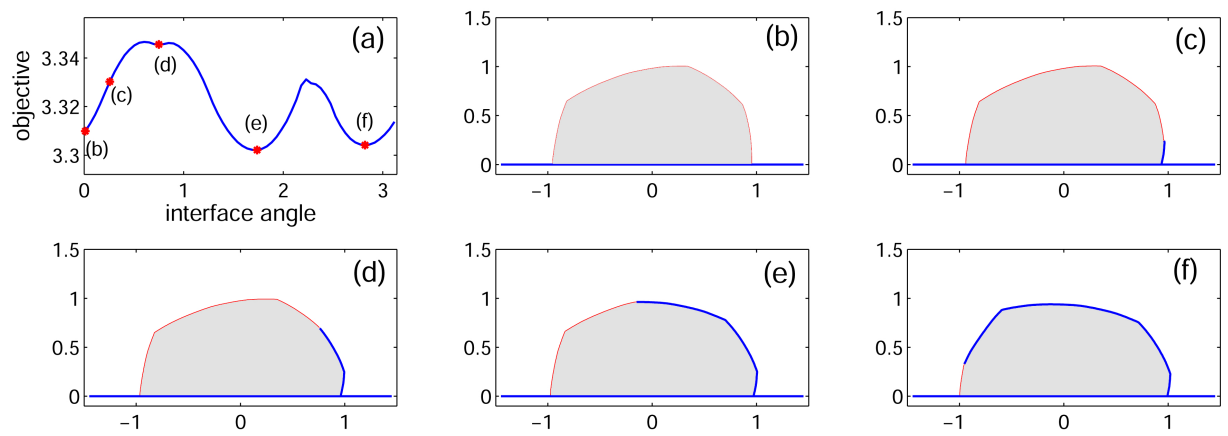

Figure 15: Two grain results: (a) shows the objective dependent on the orientation of the interface between two grains, one rotated by $\theta_{\text {rot }}=\pi / 3$ and the other one by $\theta_{\text {rot }}=\pi / 5$ from the $\operatorname{Si}(111)$ state given by Eaglesham. The indicated letters belong to the nanodots calculated in (b)-(f).

(a) the objective values are plotted against the interface angle and the letters at the markers on the curve indicate, which of the following nanodots in (b)-(f) belong to the particular values of interface angle and objective value. Interestingly one sees three local minima in (d), (e) and (f), though one would rather expect (e) and (f) to appear in experiments, as (d) is on the high plateau and seems unstable. There is another minimum for a one grain nanodot in (a) that corresponds to Figure 14 (b), while the other one-grain nanodot is located on a maximum - so that a two grain nanodot would in fact be preferred for this nanodot with this specific orientation. In (c) one example of a nanodot that is not in an equlibrated state is depicted.

The last Figure 16 shows our study for nanodots with three differently oriented phases, i.e. $\varphi_{1}=\pi / 3, \varphi_{2}=0$ and $\varphi_{3}=\pi / 5$ and we iterate the first interface over the possible positions $\theta_{1} \in[0, \pi]$ and the second one correspondingly in dependence of a fixed $\theta_{1}$ as $\theta_{2} \in\left(I_{1}, \pi\right]$. We obtain a parameter plane in $\theta_{1}$ and $\theta_{2}$. Figure 16 (a) shows the values of the surface energy corresponding to the particular positions of the interfaces in a contour plot. Four spots in the contour plots are marked and the corresponding three-grain nanodots are plotted in (b)-(e). The size of the right (blue) grain always belongs to the $\mathrm{x}$-axis in (a), the $\mathrm{y}$-axis gives the size of the middle (red) grain and if $\pi-\theta_{1}-\theta_{2}>0$ one has also the third grain with that measure in orientations. There are several extrema given in the parameter contour plot. At the axes one has a situation as in Figure 15, two grains with varying interface, and hence with different extrema already there. But also in the inner 
domain as near (b) and (c) one finds local minima. While (e) is near a minimum at the boundary (d) depicts the local maximum. The nanodot is not even convex, hence not a Winterbottom shape. We assume that if such a nanodot is formed during annealing, it destabilizes to form one of the preferred states.
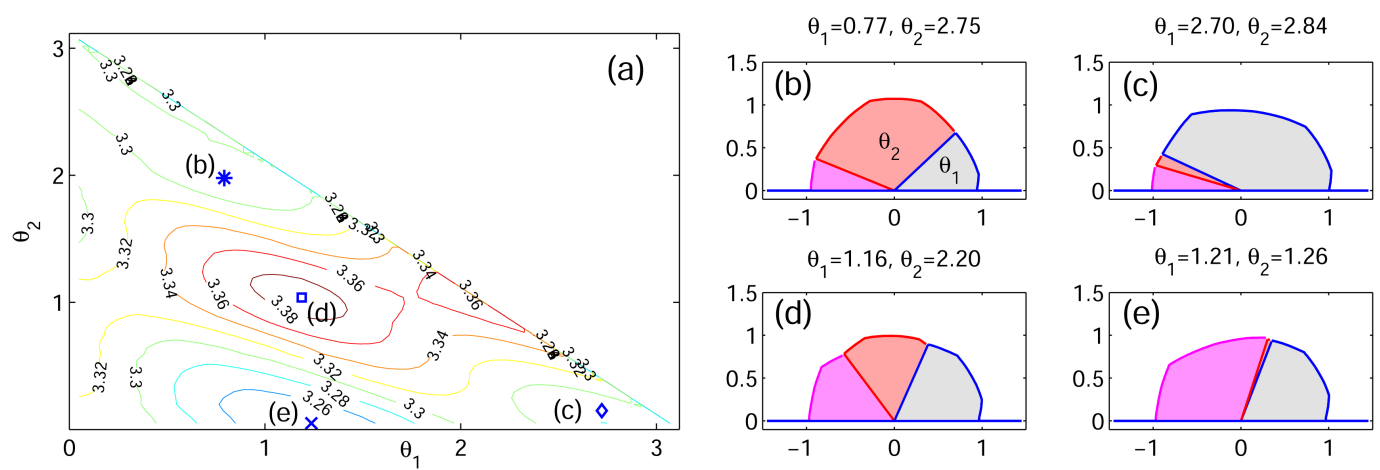

Figure 16: (a) Parameter plane: angle ranges determining the interfaces of threegrained nanodots (the angle range of third can be calculated by $\pi-\theta_{1}-\theta_{2}$ ) and the corresponding contour plot for the objective; (b)-(e) Particular nanodots corresponding to the markings in (a); (d) is in a local maximum while (b), (c) and (e) are near local minima.

\section{Conclusions and outlook}

We have introduced a new pathway to calculate equilibrium structures on a rigid substrate. We used a polar description of the interface of interest that allows also for contact angles larger than $90^{\circ}$ and discretized the integrals. Optimization with respect to the radii belonging to equidistantly distributed angles yields the desired results. The main advantage of the method is a rather straight forward way of incorporating spatial dependence into the interfacial energy $\gamma$.

Any reliable finite-dimensional constrained optimization tool seems applicable to our new formulation. We used MATLAB's fmincon package, where we typically used the SQP algorithm. It is interesting to note that the introduced numerical optimization procedure can yield the same results as the Wulff construction for the usual unbounded and adhesive case. However, for multiple grains the interface can be fixed to a certain angle of the parametrization, so that the resulting shape is not necessarily a Wulff shape. The analysis of this kind of states is interesting for a future model describing the re-crystallization/dewetting phenomenon. 
Our calculations in this study suggest that surface energy is indeed a main driving factor in the dewetting process. The differently oriented grains are mainly responsible for the asymmetric appearance of final nanodots. We have demonstrated that the problems not only have a global minimum, but also local minima corresponding to multiple-grain nanodot geometries. From the single-crystal case to the poly-crystalline setting we obtain nanodots that qualitatively mirror the experimentally observed structures. We note however that it is difficult to obtain reliable parameter values for the interfacial energies.

The long-time aim for our work is to understand and model the complete dewetting process to be able to design geometries that are optimized with respect to optical properties. The dynamics seems indispensable, in particular for realistically capturing the position of the interfaces. In our upcoming work, the insights concerning the interfacial energies for poly-crystalline nanodots derived in this study will be used for the evolution equations modeling the surface diffusion driven dewetting dynamics.

We note that it is possible to extend our method to the three-dimensional case and is part of our ongoing work, such as the incorporation of further influences that will appear as additional energy terms, such as for example elastic contributions and twinning effects, which is well-known from bulk a-Si recrystallization, see for example [2] and [3].

Finally, we note that the direct minimization approach can be used for crystalline nanodots, but also for droplets on rigid substrates and it can be easily extended, for example for droplets located on a second liquid or on nonhomogeneous substrates.

\section{Acknowledgment}

BW and MK acknowledge the financial support by the photovoltaic competence centre PVcomB.

\section{References}

[1] M. Aouassa, L. Favre, A. Ronda, H. Maaref, and I. Berbezier. The kinetics of dewetting ultra-thin Si layers from silicon dioxide. New J. Phys., 14, 2012.

[2] J. L. Batstone. In situ crystallization of amorphous silicon in the transmission electron microscope. Phil. Mag. A, 67(1):51-72, 1993. 
[3] S. Bhattacharyya, T. W. Heo, K. Chang, and L.-Q. Chen. A phase-field model of stress effect on grain boundary migration. Modelling Simul. Mater. Sci. Eng., 19:035002, 2011.

[4] K. A. Brakke. The surface evolver, http://www.susqu.edu/facstaff/b/brakke/evolver, 2003.

[5] M. Burger, F. Haußer, C. Stöcker, and A. Voigt. A Level Set Approach to Anisotropic Flows with Curvature Regularization. J. Comp. Phys., 225(1), 2007.

[6] J. W. Cahn and C. A. Handwerker. Equilibrium geometries of anisotropic surfaces and interfaces. Mat. Sci. Engin., A162:83-95, 1993.

[7] P. G. de Gennes. Wetting: statics and dynamics. Rev. Mod. Phys., 57(3):827863, 1985.

[8] D. J. Eaglesham, A. E. White, L. C. Feldman, N. Moriya, and D. C. Jacobson. Equilibrium Shape of Si. Phys. Rev. Lett., 70, 1993.

[9] C. Herring. Some theorems on the free energies of crystal surfaces. Phys. Rev., 82(1):87-93, 1951.

[10] M. D. Korzec and P. L. Evans. From bell shapes to pyramids: A reduced continuum model for self-assembled quantum dot growth. Phys. D, 239:465$474,2010$.

[11] R. V. Kutka and L. B. Freund. Minimum energy configuration of epitaxial material clusters on a lattice-mismatched substrate. J. Mech. Phys. Solids, 45, 1997.

[12] F. Leroy, F. Cheynis, T. Passanante, and P. Müller. Dynamics, anisotropy, and stability of silicon-on-insulator dewetting fronts. Phys. Rev. B, 85, 2012.

[13] E. Malguth, M. Roczen, O. Gref, A. Schoepke, and M. Schmidt. Development of ultra-thin tunneling oxides and $\mathrm{Si} / \mathrm{SiO} 2$ nanostructures for the application in silicon solar cells. Phys. Status Solidi A, 208(3):612-615, 2011.

[14] P. Miskiewicz, S. Kotarba, J. Jung, T. Marszalek, and M. Mas-Torrent. Influence of $\mathrm{SiO} 2$ surface energy on the performance of organic field effect transistors based on highly oriented, zone-cast layers of a tetrathiafulvalene derivative. J. Appl. Phys., 104, 2008. 
[15] M. Roczen, M. Schade, E. Malguth, G. Callsen, T. Barthel, O. Gref, J. A. Töfflinger, A. Schöpke, M. Schmidt, H. S. Leipner, F. Ruske, M. R. Phillips, A. Hoffmann, L. Korte, and B. Rech. Structural investigations of silicon nanostructures grown by self-organized island formation for photovoltaic applications. Appl. Phys. A, 108(3), 2012.

[16] E. J. Siem and W. C. Carter. Orientation-dependent surface tension functions for surface energy minimizing calculations. J. Mat. Sci., 40:3107-3113, 2005.

[17] E. J. Siem, W. C. Carter, and D. Chatain. The equilibrium shape of anisotropic interfacial particles. Philosophical Magazine, 84:991-1010, 2004.

[18] T. R. E. Simpson, Z. Tabatabaian, C. Jeynes, B. Parbhoo, and J. L. Keddie. Influence of Interfaces on the Rates of Crosslinking in Poly(dimethyl siloxane) Coatings. J. Poly. Sci. Part A: Poly. Chem., 42(6), 2004.

[19] B. J. Spencer. Asymptotic solutions for the equilibrium crystal shape with small corner energy regularization. Phys. Rev. E, 69:011603, 2004.

[20] W. T. Tekalign and B. J. Spencer. Evolution equation for a thin epitaxial film on a deformable substrate. J. Appl. Phys., 96(10):5505-5512, 2004.

[21] C. V. Thompson. Solid-state dewetting of thin films. Annu. Rev. Mater. Res., 42:399-434, 2012.

[22] Y. Wakayama, T. Tagami, and S. i. Tanaka. Formation of Si islands from amorphous thin films upon thermal annealing. J. Appl. Phys., 85(12), 1999.

[23] Y. Wakayama, T. Tagami, and S. i. Tanaka. Three-dimensional islands of $\mathrm{Si}$ and $\mathrm{Ge}$ formed on $\mathrm{SiO}_{2}$ through crystallization and agglomeration from amorphous thin films. Thin Solid Films, 350:300-307, 1999.

[24] A. A. Wheeler. Cahn-Hoffman $\xi$-Vector and Its Relation to Diffuse Interface Models of Phase Transitions. J. Stat. Phys., 95(5/6), 1999.

[25] W. L. Winterbottom. Equilibrium shape of a small particle in contact with a foreign substrate. Acta Metallurgica, 15:303-310, 1967.

[26] G. Wulff. Zur Frage der Geschwindigkeit des Wachstums und der Auflösung der Krystallfächen. Zeitschrift f. Krystall. Mineral., 34:449-530, 1901. 
[27] R. V. Zucker, D. Chatain, U. Dahmen, S. Hagège, and W. C. Craig. New software tools for the calculation and display of isolated and attached interfacilaenergy minimizing particle shapes. J. Mater Sci., 47:8290-8302, 2012. 